\title{
Extraction of Analytic Eigenvectors From a Parahermitian Matrix
}

\author{
Stephan Weiss ${ }^{1}$, Ian K. Proudler ${ }^{1}$, Fraser K. Coutts ${ }^{2}$, and Julian Deeks ${ }^{3}$ \\ ${ }^{1}$ Department of Electronic \& Electrical Engineering, University of Strathclyde, Glasgow G1 1XW, Scotland \\ ${ }^{2}$ Institute for Digital Communications, University of Edinburgh, Edinburgh, Scotland \\ ${ }^{3}$ DSTL Portsdown, Fareham, Hampshire PO17 6AD, UK \\ \{stephan.weiss,ian.proudler\}@strath.ac.uk, fraser.coutts@ed.ac.uk
}

\begin{abstract}
The space-time covariance matrix derived from broadband multichannel data admits - unless the data emerges from a multiplexing operation - a parahermitian matrix eigenvalue decomposition with analytic eigenvalues and analytic eigenvectors. The extraction of analytic eigenvalues has been solved previously in the discrete Fourier transform (DFT) domain; this paper addresses the approximation of analytic eigenvectors in the DFT domain. This is a two-stage process - in the first instance, we identify eigenspaces in which analytic eigenvectors can reside. This stage resolves ambiguities at frequencies where eigenvalues have algebraic mulitplicities greater than one. In a second stage, the phase ambiguity of eigenvectors is addressed by determining a maximally smooth phase response. Finally, a metric for the approximation error is derived, which allows us to increase the DFT length and iterate the two stages until a desired accuracy is reached.
\end{abstract}

\section{INTRODUCTION}

For broadband multichannel data collected by $M$ sensors in a time series vector $\mathbf{x}[n] \in \mathbb{C}^{M}$ with discrete time index $n$, second order statistics are captured by the space-time covariance matrix $\mathbf{R}[\tau]=\mathcal{E}\left\{\mathbf{x}[n] \mathbf{x}^{\mathrm{H}}[n-\tau]\right\}$, with $\mathcal{E}\{\cdot\}$ representing the expectation operator, $\{\cdot\}^{\mathrm{H}}$ the Hermitian transposition, and $\tau$ a lag parameter. This space-time covariance matrix generalises the narrowband or instantaneous covariance matrix $\mathbf{R}[0]$ to the broadband case, and leads to simple formulations of broadband problems analogous to their narrowband equivalent. To solve narrowband problems, factorisations of the covariance matrix, particularly its eigenvalue decomposition (EVD), are key. Hence, in the broadband case a similar factorisation is desired to generalise the utility of the EVD from the narrowband case.

The diagonalisation of $\mathbf{R}[\tau]$ for all lags $\tau$, or equivalently of its $z$-transform, the cross-power spectral density (CSD) $\boldsymbol{R}(z)=\sum_{\tau} \mathbf{R}[\tau] z^{-\tau}$ for all values of $\tau$, has been investigated in [1]-[3]. Since $\mathbf{R}[\tau]$ comprises auto- and cross-correlation sequences, $\boldsymbol{R}(z)$ is parahermitian, such that $\boldsymbol{R}^{\mathrm{P}}(z)=\boldsymbol{R}^{\mathrm{H}}\left(1 / z^{*}\right)=\boldsymbol{R}(z)$, where the parahermitian operator $\{\cdot\}^{\mathrm{P}}$ imposes a time reversal and Hermitian transposition. If the data $\mathbf{x}[n]$ is generated by causal and stable systems [4], then $\boldsymbol{R}(z)$ is analytic in $z$. If there is no temporal multiplexing

This work was supported by the Engineering and Physical Sciences Research Council (EPSRC) Grant number EP/S000631/1 and the MOD University Defence Research Collaboration in Signal Processing. of the data involved, then such an analytic $\boldsymbol{R}(z)$ admits a parahermitian matrix EVD (PhEVD) [2], [3],

$$
\boldsymbol{R}(z)=\mathbf{Q}(z) \boldsymbol{\Lambda}(z) \boldsymbol{Q}^{\mathrm{P}}(z),
$$

with an analytic and unitary $\boldsymbol{Q}(z)$ and analytic, diagonal, and parahermitian $\boldsymbol{\Lambda}(z)$. The diagonal elements of $\boldsymbol{\Lambda}(z)$ are referred to the analytic eigenvalues of $\boldsymbol{R}(z)$, and the columns of $\boldsymbol{Q}(z)$ their corresponding analytic eigenvectors.

In the past, a number of algorithms have targetted decompositions similar to (1). In the McWhirter or polynomial matrix EVD (PEVD) [1] with $\boldsymbol{R}(z) \approx \boldsymbol{U}(z) \boldsymbol{\Gamma}(z) \boldsymbol{U}^{\mathrm{P}}(z)$, a paraunitary matrix $\boldsymbol{U}(z)$ achieves an approximate diagonalisation with spectrally majorised eigenvalues, i.e. the latter appear strictly in descending order at every frequency. Algorithms to accomplish this, such as the second order sequential best rotation (SBR2, [1]) or the sequential matrix diagonalisation (SMD, [4]) algorithms encourage or can even be shown to converge towards spectral majorisation [5]. If diagonal elements in $\boldsymbol{\Lambda}(z)$ intersect on the unit circle, then $\boldsymbol{\Gamma}(z)$ will converge towards a non-differential and hence non-differentiable permutation of $\boldsymbol{\Lambda}(z)$. Similarly, $\boldsymbol{U}(z)$ will converge towards a permuted, and hence discontinuous version of $Q(z)$. Therefore, the factors $\boldsymbol{\Gamma}(z)$ and $\boldsymbol{U}(z)$ typically require significantly higher order polynomials for similar approximation error as achievable with analytic $\boldsymbol{\Lambda}(z)$ and $\boldsymbol{Q}(z)$.

In order to obtain lower-order approximations of the analytic factors in the PhEVD in (1), a first attempt has been reported in [6], where for a fixed DFT size, the eigenvectors where used to re-establish the coherence across bins that is otherwise lost when calculating EVDs independently in different frequency bins. To base this coherence on the eigenvectors can lead to robustness problems: eigenvectors are typically more strongly perturbed than eigenvalues [7]; in particular, at algebraic multiplicities $C>1$ of eigenvalues, the associated eigenvectors can form an arbitrary $C$-dimensional basis and hence will not necessarily yield a smooth and therefore analytic continuation of eigenvectors across these algebraic multiplicities.

Therefore, we previously have based the association of eigenvalues across frequency bins on the better conditioned eigenvalues instead of the eigenvectors [8], [9]. We use the result of this association in this paper to also extract an approximation for the analytic eigenvectors. This raises two challenges. First, continuous 1-d subspaces have to be woven 
through $C$-d subspaces at algebraic multiplicities. Secondly, the arbitrary phase function of eigenvectors must be selected as maximally smooth in order to approximate the analytic eigenvectors with a low approximation error. This second step has e.g. been addressed in [10], which uses a gradient technique driven by a smoothness metric that is to be minimised. Thus, in Sec. II, we highlight the general approach, address smooth 1-d subspaces in Sec. III and propose phase smoothing similar to [10] in Sec. IV. Finally, Sec. V assess the overall approximation error, and Sec. VI provides some results.

\section{Analytic Parahermitian Matrix Eigenvalue DECOMPOSITION AND GENERAL APPROACH}

\section{A. Properties of the PhEVD}

We assume that the space-time covariance matrix $\mathbf{R}[\tau] \circ \longrightarrow \boldsymbol{R}(z)$ is not derived from multiplexed data, such that the PhEVD of $\boldsymbol{R}(z)=\boldsymbol{Q}(z) \boldsymbol{\Lambda}(z) \boldsymbol{Q}^{\mathrm{P}}(z)$ exists with analytic factors $\boldsymbol{\Lambda}(z)$ and $\boldsymbol{Q}(z)$. From [2], [3] we know that for these factors, $\boldsymbol{\Lambda}(z)$ is unique up to a reordering. We further assume that there are no identical eigenvalues, i.e. that with $\boldsymbol{\Lambda}(z)=\operatorname{diag}\left\{\lambda_{1}(z), \ldots, \lambda_{M}(z)\right\}$, there are no functions such that $\lambda_{m}(z)=\lambda_{\mu}(z), m \neq \mu$ holds for all values of $z$ within the region of convergence. Due to the uniqueness theorem of analytic functions, $\lambda_{m}(z)$ and $\lambda_{\mu}(z)$ for $m \neq \mu$ can only share a finite number of crossing points, i.e. when evaluated on the unit circle, the eigenvalues $\lambda_{m}\left(\mathrm{e}^{\mathrm{j} \Omega}\right), m=1, \ldots, M$ will only possess algebraic multiplicities at a finite number of values $\Omega \in[0,2 \pi)$.

The matrix of eigenvectors, $\boldsymbol{Q}(z)=\left[\boldsymbol{q}_{1}(z), \ldots, \boldsymbol{q}_{m}(z)\right]$, is not uniquely defined, and each eigenvector $\boldsymbol{q}_{m}(z), m=$ $1, \ldots M$, can be modified by an arbitrary allpass function as a generalisation of the phase ambiguity of eigenvectors for the standard EVD of constant Hermitian matrices [12].

\section{B. Evaluation on the Unit Circle}

By working in the DFT domain, we evaluate $\boldsymbol{R}(z)$ in $K$ equispaced bins along the unit circle, such that ideally

$$
\boldsymbol{R}\left(\mathrm{e}^{\mathrm{j} \Omega_{k}}\right)=\boldsymbol{Q}\left(\mathrm{e}^{\mathrm{j} \Omega_{k}}\right) \boldsymbol{\Lambda}\left(\mathrm{e}^{\mathrm{j} \Omega_{k}}\right) \boldsymbol{Q}^{\mathrm{H}}\left(\mathrm{e}^{\mathrm{j} \Omega_{k}}\right)
$$

with $\Omega_{k}=\frac{2 \pi}{K} k, k=0, \ldots,(K-1)$. However, by working in isolated frequency bins, the coherence is lost, and in the $k$ th bin we obtain

$$
\boldsymbol{R}\left(\mathrm{e}^{\mathrm{j} \Omega_{k}}\right)=\mathbf{Q}_{k} \boldsymbol{\Lambda}_{k} \mathbf{Q}_{k}^{\mathrm{H}}
$$

where the r.h.s. factors are without direct equivalence to the terms on the r.h.s. of (2).

In the $k$ th bin the eigenvalues may appear randomly reordered. As a fixture, we assume the $\boldsymbol{\Lambda}_{k}$ is majorised. Therefore

$$
\boldsymbol{\Lambda}\left(\mathrm{e}^{\mathrm{j} \Omega_{k}}\right)=\mathbf{P}_{k} \mathbf{V}_{k} \boldsymbol{\Lambda}_{k} \mathbf{V}_{k}^{\mathrm{H}} \mathbf{P}_{k}^{\mathrm{T}}
$$

where $\mathbf{P}_{k}$ is a permutation matrix that reorders the otherwise spectrally majorised eigenvalues in each bin, such that they correspond to the analytic ones. The unitary $\mathbf{V}_{k}$ is a block diagonal matrix

$$
\mathbf{V}_{k}=\operatorname{diag}\left\{\mathbf{V}_{k, 1}, \ldots, \mathbf{V}_{k, L_{k}}\right\},
$$

where $L_{k}$ is the number of distinct eigenvalues in the $k$ th bin, and $\mathbf{V}_{k, \ell} \in \mathbb{C}^{Q_{k, \ell} \times Q_{k, \ell}}, \ell=1, \ldots, L_{k}$, is unitary with a dimension equivalent to the algebraic multiplicity $Q_{k, \ell}$ of the corresponding eigenvalue. Note that $\sum_{\ell=1}^{L_{k}} Q_{k, \ell}=M$. In case of $L_{k}=M$ distinct eigenvalues, $\mathbf{V}_{k}$ is a diagonal matrix imposing phase shifts only.

Therefore, for the matrix of eigenvectors, we have

$$
\mathbf{Q}\left(\mathrm{e}^{\mathrm{j} \Omega_{k}}\right)=\mathbf{Q}_{k} \mathbf{V}_{k}^{\mathrm{H}} \mathbf{P}_{k}^{\mathrm{T}} .
$$

From the extraction of analytic eigenvalues in [8], [9], we know the permutation matrices $\mathbf{P}_{k}$, and the dimensions of the unitary subblocks $\mathbf{V}_{k, \ell}$, but not their values.

\section{Problem Statement and Approach}

A number of problems remain in extracting analytic eigenvectors:

Pl (subspace alignment): In case of algebraic multiplicities, where at least some of the subblocks $\mathbf{V}_{k, \ell}$ exceed dimension one, we need to weave continuous one dimensional subspaces across these multiplicities.

$P 2$ (phase alignment): The phase shift in individual frequency bins need to be aligned to provide an overall analytic function - and therefore achieve minimum support in the time domain. P3 (approximation error): Regarding the number of frequency bins, from [8], [9] we know the value for $K$ that guarantees an extraction of eigenvalues that approximates the analytic functions with a predefined accuracy. For the approximation of eigenvectors, a different, and potentially higher number of sample points, can be required. We therefore need a criterion that can determine whether the number of sample points (and therefore the approximation order) for the eigenvectors is sufficiently high.

In contrast to the only previous algorithm for the extraction of smooth eigenvectors in [6], which addresses problem $P 2$ for a fixed and predetermined order $K$, below we tackle problems $P 1$ to $P 3$ in Secs. III to $\mathrm{V}$, respectively.

\section{Extraction of Smooth 1-D Eigenspaces}

\section{A. Rationale}

In case of a $C$-fold algebraic multiplicity of eigenvalues, with $C>1$, the corresponding eigenvectors can form an arbitrary basis within a $C$-dimensional subspace. However, to estimate analytic eigenvectors, we first need to weave smooth 1-d eigenspaces through such a manifold. Based on the assumption of non-identical eigenvalues and supported by the uniqueness theorem of analytic functions, we know that this can only occur at a finite and isolated number of frequency points.

Let $\Omega_{k_{0}}$ be a frequency bin where the eigenvalues $\lambda_{m}\left(\mathrm{e}^{\mathrm{j} \Omega_{k_{0}}}\right)=\ldots=\lambda_{m+C-1}\left(\mathrm{e}^{\mathrm{j} \Omega_{k_{0}}}\right)$ share a $C$-fold algebraic multiplicity. Since we know the analytic eigenvalues to a predefined accuracy, we can find frequency points $\Omega_{k_{0}}-\Delta$ 
and $\Omega_{k_{0}}+\Delta$ where $\Delta \ll 2 \pi / K$ and the $C$ eigenvalues are sufficiently distinct. Let $\boldsymbol{Q}_{k_{0}-}$ and $\boldsymbol{Q}_{k_{0}+}$ be the (appropriately ordered, using $\mathbf{P}_{k_{0}-}$ and $\mathbf{P}_{k_{0}+}$ ) matrices of eigenvectors for $\boldsymbol{R}\left(\mathrm{e}^{\mathrm{j}\left(\Omega_{k_{0}}-\Delta\right)}\right)$ and $\boldsymbol{R}\left(\mathrm{e}^{\mathrm{j}\left(\Omega_{k_{0}}+\Delta\right)}\right)$. We first align phases across $\boldsymbol{Q}_{k_{0}-}$ and $\boldsymbol{Q}_{k_{0}+}$, before interpolating through the appropriate $C$-dimensional subspace of $\boldsymbol{Q}_{k_{0}}$.

\section{B. Phase-Alignment Across Algebraic Multiplicities}

Let $\mathbf{q}_{m, k_{0}-}$ and $\mathbf{q}_{m, k_{0}+}$ be the $m$ th eigenvectors in the columns of $\boldsymbol{Q}_{k_{0}-}$ and $\boldsymbol{Q}_{k_{0}+}$. W.1.o.g., we retain $\boldsymbol{Q}_{k_{0}-}$ as it is, but change the $m$ th eigenvector at $\Omega_{k_{0}+\Delta}$ by a phase term $\vartheta_{m, k_{0}}$ that satisfies the optimisation problem

$$
\begin{array}{r}
\vartheta_{\mu, k_{0}, \text { opt }}=\arg \min _{\vartheta_{\mu, k_{0}}}\left\|\mathbf{q}_{\mu, k_{0}-}-\mathrm{e}^{\mathrm{j} \vartheta_{\mu, k_{0}}} \mathbf{q}_{\mu, k_{0}+}\right\|_{2}^{2}, \\
\mu=m, \cdots, m+C-1 .
\end{array}
$$

By differentiating w.r.t. $\vartheta_{\mu, k_{0}}$ and setting the gradient to zero, we obtain

$$
\vartheta_{\mu, k_{0}, \mathrm{opt}}=\arctan \frac{\operatorname{Im}\left\{\mathbf{q}_{\mu, k_{0}-}^{\mathrm{H}} \mathbf{q}_{\mu, k_{0}+}\right\}}{\operatorname{Re}\left\{\mathbf{q}_{\mu, k_{0}-}^{\mathrm{H}} \mathbf{q}_{\mu, k_{0}}+\right\}} .
$$

With these phase shift angles $\vartheta_{\mu, k_{0}, \text { opt }}, \mu=m, \ldots m+C-1$, the eigenvectors at $\Omega_{k_{0}}-\Delta$ and $\Omega_{k_{0}}+\Delta$ that are associated with the $C$-fold algebraic multiplicity in bin $k_{0}$ become aligned as closely as possible in the least squares sense.

\section{Smooth Eigenspace Selection in Algebraic Multiplicities}

Within bin $k_{0}$, there is an ambiguity of eigenvectors w.r.t. an arbitrary unitary matrix $\mathbf{V}_{k_{0}} \in \mathbb{C}^{C \times C}$, such that with eigenvectors

$$
\mathbf{U}_{k_{0}}=\left[\mathbf{q}_{m, k_{0}}, \mathbf{q}_{m+1, k_{0}}, \ldots, \mathbf{q}_{m+C-1, k_{0}}\right],
$$

the columns of $\mathbf{U}_{k_{0}}^{\prime}=\mathbf{U}_{k_{0}} \mathbf{V}_{k_{0}}^{\mathrm{H}}$ also represent valid eigenvectors. We want to solve the constrained problem

$$
\begin{gathered}
\min _{\mathbf{V}_{k_{0}}}\left\|\mathbf{V}_{k_{0}} \mathbf{U}_{k_{0}}^{\mathrm{H}} \mathbf{U}_{k_{0}-}-\mathbf{I}_{C}\right\|_{\mathrm{F}}^{2}+\left\|\mathbf{V}_{k_{0}} \mathbf{U}_{k_{0}}^{\mathrm{H}} \mathbf{U}_{k_{0}+} \boldsymbol{\Theta}_{k_{0}}-\mathbf{I}_{C}\right\|_{\mathrm{F}}^{2} \\
\text { s.t. } \quad \mathbf{V}_{k_{0}}^{\mathrm{H}} \mathbf{V}_{k_{0}}=\mathbf{I},
\end{gathered}
$$

where $\mathbf{U}_{k_{0}-} \in \mathbb{C}^{M \times C}$ and $\mathbf{U}_{k_{0}+} \in \mathbb{C}^{M \times C}$ contain the corresponding eigenvectors at the angular normalised frequencies $\Omega_{k_{0}}-\Delta$ and $\Omega_{k_{0}}+\Delta$, respectively. The diagonal matrix $\boldsymbol{\Theta}_{k_{0}}=\operatorname{diag}\left\{\mathrm{e}^{\mathrm{j} \vartheta_{m, k_{0}}}, \ldots, \mathrm{e}^{\mathrm{j} \vartheta_{m+C-1, k_{0}}}\right\}$ performs the phase alignment described in Sec. III-B. The latter is necessary such that a single unitary matrix $\mathbf{V}_{k_{0}}$ can be used to compare bin $k_{0}$ to both the preceding and subsequent bins in (10).

To solve the constrained problem (10), [13] offers a number of approaches. This requires an expression for the cost, here denoted as $\xi_{k_{0}}$, which can be written as

$$
\begin{aligned}
& \xi_{k_{0}}=\| \mathbf{V}_{k_{0}} \mathbf{U}_{k_{0}}^{\mathrm{H}} \mathbf{U}_{k_{0}-}-\mathbf{I}_{C}\left\|_{\mathrm{F}}^{2}+\right\| \mathbf{V}_{k_{0}} \mathbf{U}_{k_{0}}^{\mathrm{H}} \mathbf{U}_{k_{0}+} \boldsymbol{\Theta}_{k_{0}}-\mathbf{I}_{C} \|_{\mathrm{F}}^{2} \\
&=\operatorname{tr}\left\{\left(\mathbf{V}_{k_{0}} \mathbf{U}_{k_{0}}^{\mathrm{H}} \mathbf{U}_{k_{0}-}-\mathbf{I}_{C}\right)\left(\mathbf{V}_{k_{0}} \mathbf{U}_{k_{0}}^{\mathrm{H}} \mathbf{U}_{k_{0}-}-\mathbf{I}_{C}\right)^{\mathrm{H}}\right\} \\
&+\operatorname{tr}\left\{\left(\mathbf{V}_{k_{0}} \mathbf{U}_{k_{0}}^{\mathrm{H}} \mathbf{U}_{k_{0}+} \boldsymbol{\Theta}_{k_{0}}-\mathbf{I}_{C}\right)\right. \\
&\left.\cdot\left(\mathbf{V}_{k_{0}} \mathbf{U}_{k_{0}}^{\mathrm{H}} \mathbf{U}_{k_{0}+} \boldsymbol{\Theta}_{k_{0}}-\mathbf{I}_{C}\right)^{\mathrm{H}}\right\}
\end{aligned}
$$

Using matrix-valued differentiation [14] and Wirtinger calculus [15], we obtain as derivative $\nabla \xi_{k_{0}}=\frac{\partial \xi_{k_{0}}}{\partial \mathbf{V}_{k_{0}}}$

$$
\begin{array}{r}
\boldsymbol{\nabla} \xi_{k_{0}}=\mathbf{U}_{k_{0}}^{\mathrm{H}}\left(\mathbf{U}_{k_{0}-} \mathbf{U}_{k_{0}-}^{\mathrm{H}}+\mathbf{U}_{k_{0}+} \mathbf{U}_{k_{0}+}^{\mathrm{H}}\right) \mathbf{U}_{k_{0}} \mathbf{V}_{k_{0}}^{\mathrm{H}}- \\
-\mathbf{U}_{k_{0}}^{\mathrm{H}}\left(\mathbf{U}_{k_{0}-}+\mathbf{U}_{k_{0}+} \boldsymbol{\Theta}_{k_{0}}\right) .
\end{array}
$$

Starting from an initial guess $\mathbf{V}_{k_{0}}{ }^{(0)}$, this enables an iterative gradient search akin to [13], where in the $i$ th iteration

$$
\mathbf{V}_{k_{0}}^{(i-1 / 2)}=\mathbf{V}_{k_{0}}^{(i-1)}-\mu \nabla \xi_{k_{0}}\left(\mathbf{V}_{k_{0}}^{(i-1)}\right)
$$

is no longer necessarily a unitary matrix. Therefore, (13) alternates with a projection

$$
\mathbf{V}_{k_{0}}^{(i)}=\mathbf{\Pi}\left(\mathbf{V}_{k_{0}}^{(i-1 / 2)}\right)
$$

onto the Stiefel manifold of unitary matrices in $\mathbb{C}^{C \times C}$. The operation $\mathbf{B}=\boldsymbol{\Pi}(\mathbf{A})$ that finds the unitary matrix closest to $\mathbf{A} \in \mathbb{C}^{C \times C}$ in the least squares sense is based on the SVD $\mathbf{A}=\mathbf{U} \boldsymbol{\Sigma} \mathbf{V}^{\mathrm{H}}$; in fact we have $\boldsymbol{\Pi}(\mathbf{A})=\mathbf{U} \mathbf{V}^{\mathrm{H}}$ [12].

Thus, to solve the subspace alignment problem across algebraic multiplicities we first obtain $\boldsymbol{\Theta}_{k_{0}}$ via (8), and thereafter iterate (13) and (14) based on (12).

\section{Phase Alignment within 1-D Eigenspaces}

\section{A. Cost Function}

If $\mathbf{q}_{m, k}$ is the $k$ th bin of the $m$ eigenvector, with $m=$ $1 \ldots M$ and $\ell=0 \ldots(K-1)$, then although it now resides in a smooth eigenspace, we can still define an arbitrary phase shift $\mathrm{e}^{\mathrm{j} \varphi_{m, k}}$ for every such vector, such that $\mathbf{q}_{m, k} \mathrm{e}^{\mathrm{j} \varphi_{m, k}}$ also is a valid $m$ th eigenvector in the $k$ th frequency bin. These phase shifts must be selected such that each component of $\mathbf{q}_{m, k}$ can be interpreted as a sample point of a smooth function that is interpolated across the $K$ bins. For each of the $M$ components, $\mu=1 \ldots M$, these sample points can be organised into a vector $\mathbf{u}_{m, \mu} \in \mathbb{C}^{K}$,

$$
\mathbf{u}_{m, \mu}=\mathbf{Q}_{m, \mu} \mathbf{a}
$$

where

$$
\begin{aligned}
\mathbf{Q}_{m, \mu} & =\operatorname{diag}\left\{q_{m, \mu}[0] \ldots q_{m, \mu}[k] \ldots q_{m, \mu}[K-1]\right\} \\
\mathbf{a}_{m}^{\mathrm{T}} & =\left[\mathrm{e}^{\mathrm{j} \varphi_{m, 0}}, \ldots \mathrm{e}^{\mathrm{j} \varphi_{m, k}}, \ldots \mathrm{e}^{\mathrm{j} \varphi_{m, K-1}}\right]
\end{aligned}
$$

and $q_{m, \mu}[k]$ is the $\mu$ th component of $\mathbf{q}_{m, k}$. With these quantities, the smoothness of the interpolation of the $m$ th eigenvector - and thus the smoothness of the alignment in phase in the different bins - can be assessed by a weighted inner product which measures the power in a $p$ th derivative of the interpolation via a weighting matrix $\mathbf{C}_{p}$ defined in [11], [16]

$$
\begin{aligned}
\chi_{m} & =\sum_{\mu=1}^{M} \mathbf{u}_{m, \mu}^{\mathrm{H}} \mathbf{C}_{p} \mathbf{u}_{m, \mu} \\
& =\mathbf{a}_{m}^{\mathrm{H}} \sum_{\mu=1}^{M} \mathbf{Q}_{m, \mu}^{\mathrm{H}} \mathbf{C}_{p} \mathbf{Q}_{m, \mu} \mathbf{a}_{m}=\mathbf{a}_{m}^{\mathrm{H}} \mathbf{D}_{p, m} \mathbf{a}_{m} .
\end{aligned}
$$

The substitution $\mathbf{D}_{p, m}=\sum_{\mu=1}^{M} \mathbf{Q}_{m, \mu}^{\mathrm{H}} \mathbf{C}_{p} \mathbf{Q}_{m, \mu}$ permits to express this smoothness metric directly in terms of the vector of phase shifts $\mathbf{a}_{m}$ for the $m$ th eigenvector. 


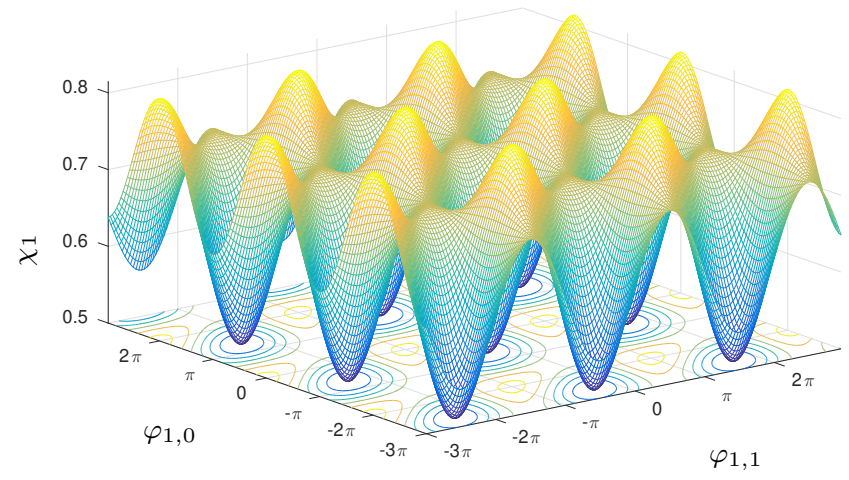

Fig. 1. Cost function value $\chi_{1}$ in dependency of $\varphi_{1,0}$ and $\varphi_{1,1}$.

\section{B. Gradient-Based Iterative Optimisation}

The minimisation of $\chi_{m}$ is accomplished by appropriately tuning the phase in every frequency bin, assembled into a vector $\varphi_{m}=\left[\varphi_{m, 0}, \ldots, \varphi_{m, K-1}\right]^{\mathrm{T}} \in \mathbb{R}^{K}$,

$$
\boldsymbol{\varphi}_{m, \mathrm{opt}}=\arg \min _{\boldsymbol{\varphi}_{m}} \mathbf{a}_{m}^{\mathrm{H}} \mathbf{D}_{p, m} \mathbf{a}_{m}=\arg \min _{\boldsymbol{\varphi}_{m}} \chi_{m} .
$$

An example of the cost function $\chi_{1}$ for $M=2$ and

$$
\left[\boldsymbol{q}_{1}(z) \boldsymbol{q}_{2}(z)\right]=\frac{1}{2}\left[\begin{array}{ll}
1+z^{-1} & 1-z^{-1} \\
1-z^{-1} & 1+z^{-1}
\end{array}\right]
$$

is shown in Fig. 1 in dependency on phase shifts in the two frequency bins for $K=2$. Since this cost function appears difficult to solve in closed form, we attempt an iterative gradient approach, such that $\varphi_{m}$ depends on an iteration index $n$, and

$$
\boldsymbol{\varphi}_{m}[n+1]=\boldsymbol{\varphi}_{m}[n]-\mu \frac{\partial \chi_{m}}{\partial \boldsymbol{\varphi}_{m}} .
$$

Since $a_{m}[k]=\mathrm{e}^{\mathrm{j} \varphi_{m, k}}$, we can define

$$
\begin{aligned}
\frac{\partial}{\partial \boldsymbol{\varphi}_{m}} & =\frac{\partial \mathbf{a}_{m}^{\mathrm{H}}}{\partial \boldsymbol{\varphi}_{m}} \frac{\partial}{\partial \mathbf{a}_{m}^{*}}+\frac{\partial \mathbf{a}_{m}^{\mathrm{T}}}{\partial \boldsymbol{\varphi}_{m}} \frac{\partial}{\partial \mathbf{a}_{m}} \\
& =-\mathrm{j} \boldsymbol{\Phi}_{m}^{*} \frac{\partial}{\partial \mathbf{a}_{m}^{*}}+\mathrm{j} \boldsymbol{\Phi}_{m} \frac{\partial}{\partial \mathbf{a}_{m}}
\end{aligned}
$$

with

$$
\boldsymbol{\Phi}_{m}=\operatorname{diag}\left\{\mathrm{e}^{\mathrm{j} \varphi_{m, 0}}, \ldots, \mathrm{e}^{\mathrm{j} \varphi_{m, L-1}}\right\} .
$$

Note that $\boldsymbol{\Phi}_{m} \underline{1}=\mathbf{a}_{m}$. Therefore

$$
\begin{aligned}
\frac{\partial \chi_{m}}{\partial \boldsymbol{\varphi}_{m}} & =-\mathrm{j} \boldsymbol{\Phi}_{m}^{*} \mathbf{D}_{p, m} \mathbf{a}_{m}+\mathrm{j} \boldsymbol{\Phi}_{m} \mathbf{D}_{p, m}^{\mathrm{T}} \mathbf{a}_{m}^{*} \\
& =2 \operatorname{Im}\left\{\boldsymbol{\Phi}_{m}^{*} \mathbf{D}_{p, m} \boldsymbol{\Phi}_{m} \underline{1}\right\} .
\end{aligned}
$$

This defines the iterative update scheme.

\section{Fast Newton Approach}

The convergence of the iterative approach in (22) can be enhanced using a fast Newton approach [17] based on

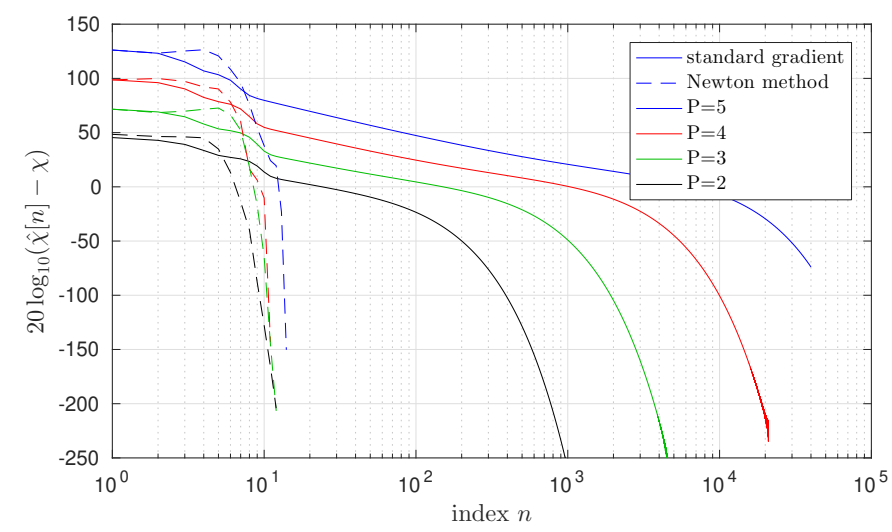

Fig. 2. Convergence curves for phase adaptation with different power of derivatives considered.

the Hessian matrix $\mathbf{H}=2 \operatorname{Re}\left\{\boldsymbol{\Phi}_{m}^{*} \mathbf{D} \boldsymbol{\Phi}_{m}\right\}$ derived similarly to [10]. The resulting Newton update $\mathbf{H}^{-1}$, such that

$$
\boldsymbol{\varphi}_{m}[n+1]=\boldsymbol{\varphi}_{m}[n]-\mu \mathbf{H}^{-1} \frac{\partial \chi_{m}}{\partial \boldsymbol{\varphi}_{m}} .
$$

Example. For a $K=64$-point DFT of $\mathbf{q}_{1}[n] \circ-\bullet \boldsymbol{q}_{1}(z)$ in (21), the phase is altered randomly. Thereafter, the above iterative updating schemes are used to calculate the phase. The results against the optimum attainable smoothness metric of $\chi=\frac{1}{2}$ for an undisturbed phase are shown in Fig. 2 . The iterative gradient algorithm in (22) converges slowly, particularly if the power in the derivatives are taken at higherorder, such as $p=5$, with faster adaptation for lower $p$. In constrast, the fast Newton update significantly reduces the number of iterations, reaching machine precision after around 12 iterations as seen in Fig. 2.

While the update (28) can converge faster when the term $\mathbf{H}^{-1}$ is included, it may be attracted to critical points [13]. From Fig. 1 we know that the cost function can include saddle points and local minima. This can cause problems, which can be overcome by a modulation applied to $\boldsymbol{\Phi}_{m}$ after initial convergence, and re-checking whether an enhanced solution is attained. For lack of space, this is not further elaborated here.

\section{APPROXIMATION ORDER AND APPROXIMATION ERROR}

Any approximation error will make itself felt by a lack of paraunitarity. While the eigenvectors $\boldsymbol{q}_{m}\left(\mathrm{e}^{\mathrm{j} \Omega}\right), m=1 \ldots M$, are orthogonal at the sample points $\Omega=\Omega_{k}=2 \pi k / K, k=$ $0 \ldots(K-1)$, and therefore $\mathbf{Q}_{k}=\left[\boldsymbol{q}_{1}\left(\mathrm{e}^{\mathrm{j} \Omega_{k}}\right), \ldots, \boldsymbol{q}_{M}\left(\mathrm{e}^{\mathrm{j} \Omega_{k}}\right)\right]$ unitary, the continuous trigonometric or Dirichlet interpolations $\hat{\mathbf{Q}}\left(\mathrm{e}^{\mathrm{j} \Omega}\right)$ through $\mathbf{Q}_{k}, k=0, \ldots,(K-1)$ may not satisfy unitarity. The interpolation $\hat{\mathbf{Q}}\left(\mathrm{e}^{\mathrm{j} \Omega}\right)$ will only be unitary for all $\Omega \in \mathbb{R}$ if the arbitrary phase at every frequency value does not affect orthogonality. We can measure therefore

$$
\epsilon_{K}=\frac{1}{2 \pi} \int_{-\pi}^{\pi}\left\|\hat{\boldsymbol{Q}}\left(\mathrm{e}^{\mathrm{j} \Omega}\right) \hat{\boldsymbol{Q}}^{\mathrm{H}}\left(\mathrm{e}^{\mathrm{j} \Omega}\right)-\mathbf{I}\right\|_{\mathrm{F}}^{2} \mathrm{~d} \Omega,
$$

where $\epsilon_{K}$ is the error linked to the approximation order $K$. 
To evaluate $\epsilon_{K}$, we expand (29), to obtain the trigonometric interpolation $\hat{\boldsymbol{q}}_{\mu}\left(\mathrm{e}^{\mathrm{j} \Omega}\right)$ of $\boldsymbol{q}_{m}\left(\mathrm{e}^{\mathrm{j} \Omega_{k}}\right)$,

$$
\begin{array}{r}
\epsilon_{K}=\frac{1}{2 \pi} \int_{-\pi}^{\pi} \sum_{m=1}^{M}\left(\sum_{\mu=1}^{M}\left|\hat{\boldsymbol{q}}_{m}^{\mathrm{H}}\left(\mathrm{e}^{\mathrm{j} \Omega}\right) \hat{\boldsymbol{q}}_{\mu}\left(\mathrm{e}^{\mathrm{j} \Omega}\right)\right|^{2}-\right. \\
\left.-2 \hat{\boldsymbol{q}}_{m}^{\mathrm{H}}\left(\mathrm{e}^{\mathrm{j} \Omega}\right) \hat{\boldsymbol{q}}_{m}\left(\mathrm{e}^{\mathrm{j} \Omega}\right)+1\right) \mathrm{d} \Omega .
\end{array}
$$

For the second term in (30) we have based on Parseval

$$
\frac{1}{2 \pi} \int_{-\pi}^{\pi} \sum_{m=1}^{M} \hat{\boldsymbol{q}}_{m}^{\mathrm{H}}\left(\mathrm{e}^{\mathrm{j} \Omega}\right) \hat{\boldsymbol{q}}_{m}\left(\mathrm{e}^{\mathrm{j} \Omega}\right) \mathrm{d} \Omega=\frac{1}{K} \sum_{k=0}^{K-1}\left\|\mathbf{Q}_{k}\right\|_{\mathrm{F}}^{2}=M .
$$

For the first term in (30),

$$
\begin{aligned}
\frac{1}{2 \pi} \int_{-\pi}^{\pi} \sum_{m=1}^{M}\left(\sum_{\mu=1}^{M}\left|\hat{\boldsymbol{q}}_{m}^{\mathrm{H}}\left(\mathrm{e}^{\mathrm{j} \Omega}\right) \hat{\boldsymbol{q}}_{\mu}\left(\mathrm{e}^{\mathrm{j} \Omega}\right)\right|^{2} \mathrm{~d} \Omega=\right. & =\frac{1}{2 K} \sum_{\kappa=0}^{2 K-1}\left\|\hat{\mathbf{O}}_{\kappa}^{\mathrm{H}} \hat{\mathbf{O}}_{\kappa}\right\|_{\mathrm{F}}^{2},
\end{aligned}
$$

where $\hat{\mathbf{O}}_{\kappa}$ is a twofold oversampled version of $\mathbf{Q}_{k}$, with a trigonometric interpolation such that for $\kappa \in \mathbb{Z}$

$$
\hat{\mathbf{O}}_{2 \kappa}=\mathbf{Q}_{\kappa} \quad \text { and } \quad \hat{\mathbf{O}}_{2 \kappa+1}=\sum_{k=0}^{K-1} \alpha_{k} \mathbf{Q}_{\kappa+k+1} .
$$

Working with sample points on the unit circle implies a cyclic wrap-around of the coefficients $\mathbf{Q}_{k}$ for $k \geq K$. It can be shown that the interpolation filter possesses the coefficients of a Dirichlet kernel for even $K$ [18].

Since the $\mathbf{Q}_{k}$ are unitary, $\left\|\mathbf{Q}_{k}\right\|_{\mathrm{F}}^{2}=M$. Therefore, (31) can be split into two sums, one for odd and one for even values of $\kappa$, and the cost $\epsilon_{K}$ simplifies to

$$
\epsilon_{K}=\frac{1}{K} \sum_{\kappa=0}^{K-1}\left\|\sum_{k=0}^{K} \alpha_{k} \mathbf{Q}_{\kappa+k+1}\right\|_{\mathrm{F}}^{2}-M .
$$

Thus, the error in paraunitarity can be precisely measured from a simple two-fold trigonometric interpolation of the sample points, and if required, $K$ can be doubled with a repeat of $P I$ and $P 2$ until a desired accuracy has been reached.

\section{Simulation AND Results}

As an example to simulate the proposed approach, we build $\boldsymbol{R}(z)$ from the example in [2] with $\boldsymbol{\Lambda}(z)=$ $\operatorname{diag}\left\{z+3+z^{-1}, \mathrm{j} z+3-\mathrm{j} z^{-1}\right\}$. With $\boldsymbol{q}_{1}(z)$ and $\boldsymbol{q}_{2}(z)$ from (21), we perturb a sampled version of $\boldsymbol{Q}\left(\mathrm{e}^{\mathrm{j} \Omega}\right)$ for $K=8$ via

$$
\mathbf{Q}_{k}=\mathbf{Q}\left(\mathrm{e}^{\mathrm{j} \Omega_{k}}\right) \operatorname{diag}\left\{\mathrm{e}^{\mathrm{j} \psi_{1, k}}, \mathrm{e}^{\mathrm{j} \psi_{2, k}}\right\} \mathbf{V}_{k}
$$

with random, uniformly distributed angles $\psi_{m, k}, m=1,2$, $k=0, \ldots(K-1)$. Initially, $\mathbf{V}_{k}=\mathbf{I} \forall k$. For an ensemble of 1000 randomisations of $\left\{\psi_{m, k}\right\}$, we recover $\hat{\boldsymbol{Q}}(z)$ with an average $\epsilon_{K}=2.54 \mathrm{e}-16 \pm 2.67 \mathrm{e}-15$ using the proposed approach or equivalently the methods in [6], [10].

If additionally a random unitary subspace perturbation $\mathbf{V}_{k}$ occurs at the algebraic multiplicities of $\Omega_{1}=\frac{\pi}{4}$ and $\Omega_{5}=\frac{\pi}{5}$, then [6] fails to recover eigenvalues and eigenvectors independent of how large $K$ is selected. In the proposed approach, the eigenvalues can be recovered using [8], [9], and with $K=8$ and $\Delta=2^{-8} \frac{2 \pi}{K}$ in $P 1$, the proposed method finds the correct eigenvectors with $\epsilon_{K}=5.78 \mathrm{e}-13 \pm 1.83 \mathrm{e}-11$. Because the ground truth has finite order, no iteration via $P 3$ is required.

\section{CONCLUSION}

This paper has targetted a method for extracting approximations of analytic eigenvectors from analytic parahermitian matrices. Operating in the DFT domain, this requires (i) to re-establish coherence and find the correct association across frequency bins. This is a byproduct of the previous extraction of analytic eigenvalues in [8], [9], which is robust w.r.t. algebraic multiplicities of eigenvalues greater than one. Thus, the proposed approach concentrates on (ii) subspace and (iii) phase alignment of eigenvectors across bins. Particularly (ii) adds robustness over existing approaches in [6], [10]. Additionally, a simple error metric in the DFT domain allows us to iterate the approach by increasing the DFT length until a sufficient accuracy has been reached.

\section{REFERENCES}

[1] J.G. McWhirter, P. D. Baxter, T. Cooper, S. Redif, and J. Foster, "An EVD Algorithm for Para-Hermitian Polynomial Matrices," IEEE Trans. SP, 55(5):2158-2169, May 2007.

[2] S. Weiss, J. Pestana, and I.K. Proudler, "On the existence and uniqueness of the eigenvalue decomposition of a parahermitian matrix," IEEE Trans. SP, 66(10):2659-2672, May 2018.

[3] S. Weiss, J. Pestana, I.K Proudler, and F.K. Coutts, "Corrections to "on the existence and uniqueness of the eigenvalue decomposition of a parahermitian matrix"," IEEE Trans. SP, 66(23):6325-6327, Dec. 2018.

[4] S. Redif, S. Weiss, and J.G. McWhirter, "Sequential matrix diagonalization algorithms for polynomial EVD of parahermitian matrices," IEEE Trans. SP, 63(1):81-89, Jan. 2015.

[5] J. G. McWhirter and Z. Wang, "A novel insight to the SBR2 algorithm for diagonalising para-hermitian matrices," in 11th IMA Maths in Signal Proc., Birmingham, UK, Dec. 2016.

[6] M. Tohidian, H. Amindavar, and A. M. Reza, "A DFT-based approximate eigenvalue and singular value decomposition of polynomial matrices," EURASIP J. Advances in Signal Proc., 2013(1):1-16, 2013.

[7] C. Delaosa, J. Pestana, N.J. Goddard, S. Somasundaram, and S. Weiss, "Sample space-time covariance matrix estimation," in IEEE ICASSP, pp. 8033-8037, Brighton, UK, May 2019.

[8] S. Weiss, I.K. Proudler, F.K. Coutts, and J. Pestana, "Iterative approximation of analytic eigenvalues of a parahermitian matrix EVD," in IEEE ICASSP, Brighton, UK, May 2019.

[9] S. Weiss, I.K. Proudler, and F.K. Coutts, "Eigenvalue decomposition of a parahermitian matrix: extraction of analytic eigenvalues," IEEE Trans. SP, submitted, 2020.

[10] F.K. Coutts, K. Thompson, J. Pestana, I.K. Proudler, and S. Weiss, "Enforcing eigenvector smoothness for a compact DFT-based polynomial eigenvalue decomposition," in IEEE SAM, Sheffield, UK, July 2018.

[11] S. Weiss, J. Selva, and M. Macleod, "Measuring smoothness of trigonometric interpolation through incomplete sample points," in EUSIPCO, Amsterdam, The Netherlands, Jan. 2021.

[12] G.H. Golub and C.F. Van Loan, Matrix Computations, 3rd ed. Baltimore, Maryland: John Hopkins University Press, 1996.

[13] J. H. Manton, "Optimization algorithms exploiting unitary constraints,' IEEE Trans. SP, 50(3):635-650, Mar. 2002.

[14] A. Hjørungnes and D. Gesbert, "Complex-valued matrix differentiation: Techniques and key results," IEEE TSP, 55(6):2740-2746, June 2007.

[15] W. Wirtinger, "Zur formalen Theorie der Funktionen von mehr komplexen Veränderlichen,” Mathematische Annalen, 97:357 - 376, 1926.

[16] S. Weiss and M. D. Macleod, "Maximally smooth dirichlet interpolation from complete and incomplete sample points on the unit circle," in IEEE ICASSP, Brighton, UK, May 2019.

[17] S. Haykin, Adaptive Filter Theory, 2nd ed, Prentice Hall, 1991.

[18] A. Zygmund, Trigonometric Series, 2nd ed, Cambridge Uni. Press, 1959. 